\title{
Evolução da função renal de pacientes portadores do Vírus da Imunodeficiência Humana/ Síndrome da Imunodeficiência Adquirida*
}

\author{
Renal function in patients with Human Immunodeficiency Virus
}

Evolución de la función renal de pacientes portadores del Virus de la Inmunodeficiencia Humanal

Sindrome de la Inmunodeficiencia Adquirida

\section{Daniane Bornea Friedl1, Daniele Cristina Bosco Aprile ${ }^{2}$, Luana Camargo Fino', Dulce Aparecida Barbosa ${ }^{3}$, Angélica Gonçalves Silva Belasco ${ }^{3}$}

\begin{abstract}
RESUMO
Objetivo: Avaliar a evolução da função renal em pacientes portadores do Vírus da Imunodeficiência Humana que iniciaram acompanhamento no Centro de Controle de Doenças Infecciosas do Hospital São Paulo/ Universidade Federal de São Paulo. Métodos: Estudo retrospectivo realizado através da análise de 200 prontuários selecionados de forma aleatória. Resultados: Perfil predominante masculino, cor branca, idade média de 45 anos, mais de 50 meses de diagnóstico, co-morbidades adquiridas variando de zero a nove doenças, creatinina média de $0,93 \mathrm{mg} / \mathrm{dl}$ e a grande maioria realizando tratamento medicamentoso. Conclusão: O grupo analisado não mostrou alteração significativa em relação à função renal entre a primeira e a última consulta.
\end{abstract}

Descritores: HIV; Insuficiência renal crônica; Co-morbidades; Anti-retrovirais

\begin{abstract}
Objective: To evaluate the renal function in patients with Human Immunodeficiency Virus who were attending the Center for Infectious Diseases Control of the São Paulo Hospital of the Federal University of São Paulo. Methods: This retrospective study consisted of the review of 200 randomly selected medical records. Results: Patients were predominant white males with a mean age of 45 years. They had been diagnosed with the disease over 50 months ago, had from 0 to up 9 comorbidities, and a mean creatinine of $.93 \mathrm{mg} / \mathrm{dl}$. The majority of patients were receiving antiviral medication. Conclusion: There was no significant alteration in renal function in the patients between the first and last visit.
\end{abstract}

Keywords: HIV; Renal insufficiency, Chronic; Comorbidity; Anti-retroviral agents

\section{RESUMEN}

Objetivo: Evaluar la evolución de la función renal en pacientes portadores del Virus de la Inmunodeficiencia Humana que iniciaron acompañamiento en el Centro de Control de Enfermedades Infecciosas del Hospital Sao Paulo/ Universidad Federal de Sao Paulo. Métodos: Estudio retrospectivo realizado a través del análisis de 200 historias clínicas seleccionadas de forma aleatoria. Resultados: Perfil predominante masculino, raza blanca, edad promedio de 45 años, más de 50 meses de diagnóstico, comorbidades adquiridas variando de cero a nueve enfermedades, creatinina promedio de $0,93 \mathrm{mg} / \mathrm{dl}$ y la gran mayoría realizando tratamiento medicamentoso. Conclusión: El grupo analizado no mostró alteración significativa en relación a la función renal entre la primera y la última consulta.

Descritores: VIH; Insuficiencia renal crônica; Comorbilidad; Agentes antirretrovirales

\footnotetext{
${ }^{*}$ Estudo realizado no Centro de Controle de Doenças Infecciosas do Hospital São Paulo, Universidade Federal de São Paulo - UNIFESP (SP), Brasil.

${ }^{1}$ Enfermeira graduada pela Universidade Federal de São Paulo - UNIFESP - São Paulo (SP), Brasil.

${ }^{2}$ Pós-graduando (Mestrado) em Ciências pela Faculdade de Medicina da Universidade de São Paulo - USP - São Paulo (SP), Brasil.

${ }^{3}$ Pós-Doutor em Nefrologia, Professora Adjunto da Universidade Federal de São Paulo - UNIFESP - São Paulo (SP), Brasil;
} 


\section{INTRODUÇÃO}

Atualmente, estima-se que existam 33,2 milhões de pessoas com o Vírus da Imunodeficiência Humana (HIV) no mundo e que ocorreram 2,5 milhões de novas infecções e 2,1 milhões de óbitos em 2007. No Brasil, de 1980 a junho de 2007 , foram notificados 474.273 casos, com taxa de prevalência da infecção pelo HIV de $0,6 \%$ na população de 15 a $49 \operatorname{anos}^{(1)}$.

A incidência da Síndrome da Imunodeficiência Adquirida (SIDA) no Brasil indica mudança na sua distribuição, com tendência à feminização, interiorização, heterossexualização, pauperização e baixa escolaridade ${ }^{(2)}$. Há também queda na letalidade no país, desde 1995, com a adoção da terapia antirretroviral (HAART) ${ }^{(3)}$.

A insuficiência renal crônica (IRC) consiste na deterioração irreversível e progressiva da função renal ${ }^{(4)}$. A IRC também é definida de acordo com o Rítmo de Filtração Glomerular, abaixo de $60 \mathrm{~mL} / \mathrm{min} / 1.73 \mathrm{~m}^{2}$, com ou sem lesão renal ${ }^{(5)}$.

A prevalência de afecções renais está aumentando na população infectada pelo HIV. Em março de 2008 a prevalência de sorologia positiva para HIV em pacientes em diálise crônica no Brasil era de $0,7 \%{ }^{(4)}$. Provavelmente, isso se deve, a um reflexo do aumento das afecções renais na população geral não infectada, já que fatores de risco como diabetes mellitus e hipertensão arterial têm aumentado nesta população ${ }^{(6-7)}$. Alguns autores apontam $\mathrm{CD}^{+}$(linfócitos $\mathrm{TCD}^{+}$) baixo, carga viral alta e raça negra como fatores de risco para o desenvolvimento de HIV-associated nephropathy (HIVAN) ${ }^{(6)}$.

Muitas drogas utilizadas para o tratamento de pacientes são nefrotóxicas ${ }^{(6,8)}$. Indinavir, Tenofovir, Sulfadiazina, Pentamidina e Sulfametoxazole Trimetoprima são medicamentos com provável relação com o desenvolvimento de nefropatias ${ }^{(()}$.

O tratamento para controle dos portadores do HIV/ AIDS está se mostrando mais eficaz e, com isso, observase o aumento da sobrevida e da incidência de HIVAN. O objetivo deste estudo foi avaliar a evolução da função renal em pacientes portadores do HIV, que iniciaram acompanhamento no Centro de Controle de Doenças Infecciosas do Hospital São Paulo/ Universidade Federal de São Paulo.

\section{MÉTODOS}

Este estudo é do tipo retrospectivo descritivo, com dados obtidos em prontuários de pacientes com acompanhamento ambulatorial no Centro de Controle de Doenças Infecciosas do Hospital São Paulo, Universidade Federal de São Paulo - UNIFESP.

O período da coleta de dados ocorreu de outubro de 2005 a março de 2008. O número total de prontuários era de 1006 e desses foram avaliados 200. O levantamento dos mesmos foi aleatório, junto ao Serviço de Arquivo Médico desse Hospital. Os dados levantados foram: sóciodemográficos, tempo de diagnóstico, comorbidades, tratamento medicamentoso e exames laboratoriais. Após a coleta desses dados foi realizado o cálculo da estimativa da Filtração Glomerular, Clearance de Creatinina ( $\mathrm{ml} / \mathrm{min}$ ), e do ritmo de filtração glomerular $\left(\mathrm{ml} / \mathrm{min} / 1,73 \mathrm{~m}^{2}\right)$.

A equação de Cockcroft-Gault é a fórmula mais usada para estimar a Taxa de Filtração Glomerular (TFG) em adultos ${ }^{(4)}$. Ela foi desenvolvida para estimar a depuração da creatinina e, por isso, estima a TFG. Utilizou-se também a equação abreviada do estudo de Modification of Diet in Renal Disease.

Considerou-se, neste estudo, creatinina elevada quando maior que $1,2 \mathrm{mg} / \mathrm{dL}$, conforme estabelecido pelo laboratório do Hospital São Paulo, para indicar alteração renal.

Este estudo foi aprovado pelo Comitê de Ética em Pesquisa da UNIFESP, registrado sob o número 1358/05.

\section{RESULTADOS}

Os resultados do estudo são mostrados nas Tabelas 1 e 2 e Figura 1 e as variáveis contínuas são expressas em média \pm desvio padrão, variação e percentuais.

Tabela 1 - Características sóciodemográficas e relacionadas à doença de pacientes com HIV/AIDS

\begin{tabular}{lc}
\hline Características & Pacientes (n=200) \\
\hline Média de idade (anos) \pm dp & $44,6 \pm 8,3$ \\
Sexo (\%) & \\
Masculino & 58,5 \\
Feminino & 41,5 \\
Opção sexual (\%) & \\
Heterossexuais & 66,0 \\
Homossexuais & 14,5 \\
Bissexuais & 5,5 \\
Sem dados & 14,0 \\
Cor de pele (\%) & \\
Branca & 46,5 \\
Negra & 17,5 \\
Parda & 33,0 \\
Sem dados & 3,0 \\
Tempo de diagnóstico meses (\%) & \\
$<50$ & 26,5 \\
50 -100 & 29,5 \\
$>100$ & 40,0 \\
Sem dados & 4,0 \\
Tratamento medicamentoso (\%) & 87,0 \\
Realizado & 7,5 \\
Não realizado & 5,5 \\
Sem dados & $2,7(0-9)$ \\
Co-morbidades - média (variação) & \\
\hline
\end{tabular}


Tabela 2 - Exames laboratoriais de portadores de HIV/ AIDS (média da primeira dosagem versus média da última dosagem durante o tempo de acompanhamento no serviço)

\begin{tabular}{lcc}
\hline Exames & $\begin{array}{c}\text { Primeira } \\
\text { dosagem }\end{array}$ & $\begin{array}{c}\text { Última } \\
\text { dosagem }\end{array}$ \\
\hline TFG $\left(\mathrm{ml} / \mathrm{min} / 1,73 \mathrm{~m}^{2}\right)$ & 104,3 & 99,37 \\
Sem dados & $11(5,5 \%)$ & $12(6 \%)$ \\
Creatinina sérica $(\mathrm{mg} / \mathrm{dL})$ & 0,93 & 0,93 \\
Variação & $0,3-2,4$ & $0,4-1,9$ \\
Sem dados & $4(2 \%)$ & $4(2 \%)$ \\
Clearance Creat. (mL/min) & 102 & 100 \\
Sem dados & $17(8,5 \%)$ & $22(11 \%)$ \\
HDL mg/dL & 39,3 & 45,9 \\
Sem dados & $46(23 \%)$ & $20(10 \%)$ \\
LDL mg/dL & 102,2 & 113 \\
Sem Dados & $53(26,5 \%)$ & $28(14 \%)$ \\
Triglicérides mg/dL & 184,2 & 201,6 \\
Sem dados & $50(25 \%)$ & $22(11 \%)$ \\
Colesterol total mg/dL & 178,8 & 198,6 \\
Sem dados & $43(21,5 \%)$ & $20(10 \%)$ \\
CD4+ (cel/mm $)$ & 346,2 & 481,6 \\
Sem dados & $17(8,5 \%)$ & $6(3 \%)$ \\
CD ${ }^{+}$(cel/mm m $\left.^{3}\right)$ & 1004,3 & 937,8 \\
Sem dados & $31(15,5 \%)$ & $9(4,5 \%)$ \\
Carga viral & 489998 & 31346 \\
Sem dados & $51(25,5 \%)$ & $33(16,5 \%)$ \\
\hline
\end{tabular}

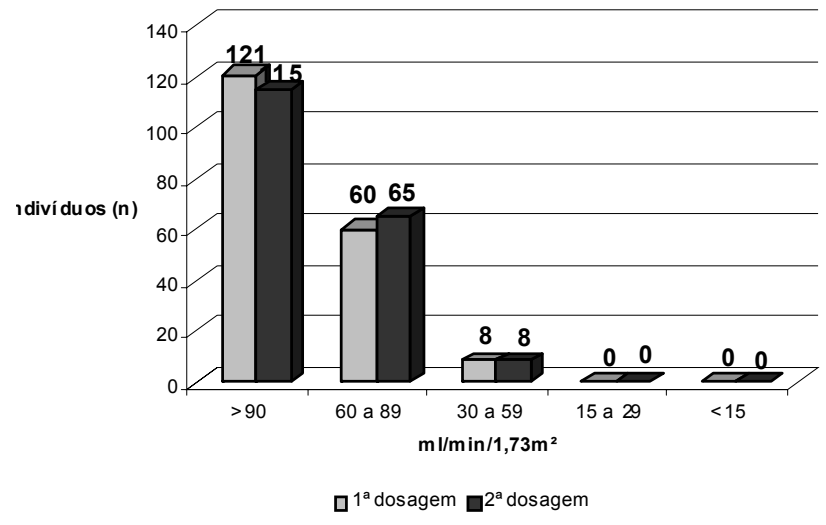

Figura 1 - Alterações da Taxa de Filtração Glomerular em pacientes com HIV/AIDS

\section{DISCUSSÃO}

A maioria da população estudada foi masculina, idade média de 45 anos, branca, mais de 50 meses de diagnóstico, média de três co-morbidades adquiridas variando de zero a nove doenças e 174 (87\%) realizavam o tratamento medicamentoso.

Houve predominância de heterossexuais e relativo equilíbrio entre a população masculina e feminina, de acordo com dados da literatura, cujo perfil atual mostra a heterossexualização e a feminização da evolução da doença $a^{(1-2)}$.

Pacientes assintomáticos que faziam uso de HAART não desenvolveram HIVAN, indicando que a terapia pode ser um fator renoprotetor para pacientes infectados assintomáticos. As terapias antirretrovirais podem ser usadas como tratamento e prevenção de HIVAN ${ }^{(6)}$. Neste estudo, oito pacientes com TFG abaixo de $60 \mathrm{ml} / \mathrm{min} /$ $1,73 \mathrm{~m}^{2}$ na $1^{\text {a }}$ dosagem, apresentaram aumento na TFG na $2^{\mathrm{a}}$ dosagem, conforme Figura 1. Esses pacientes estavam em uso de HAART.

Em relação às co-morbidades mais prevalentes entre os pacientes estudados, encontrou-se diabetes mellitus, hipertensão arterial e hepatite $C$, que são consideradas fatores de risco para o desenvolvimento de nefropatias, principalmente na população portadora de HIV com baixo e carga viral alta ${ }^{(6-8)} \mathrm{CD} 4^{+}$. Além dessas, neste estudo, a dislipidemia foi o problema mais freqüente, sendo fator de risco para doenças cardiovasculares, desencadeantes $\mathrm{e} / \mathrm{ou}$ agravantes e/ou conseqüentes de nefropatias ${ }^{(0-10)}$. Estudos recentes mostram importantes alterações metabólicas em pacientes infectados pelo HIV que fazem uso de HAART, como síndrome lipodistrófica ${ }^{(11)}$. Os valores médios de LDL e HDL (Low and High Density Lipoproteins) aumentaram, porém, se mantiveram dentro da normalidade. Houve aumento da média do colesterol total para um valor limítrofe e o triglicéride passou de um valor limítrofe para um valor elevado.

O valor médio do CD4+ aumentou enquanto o de CD8+ (Linfócitos $\mathrm{T}^{-\mathrm{CD} 8} 8^{+}$) e a carga viral apresentaram redução.

Dos 174 (86,5\%) pacientes que utilizaram algum tipo de medicamento, 108 (62\%) usaram alguma droga nefrotóxica: $66(61,1 \%)$ Sulfametoxazole Trimetoprima; $21(19,4 \%)$ Tenofovir; 15 (14\%) Indinavir e 6 (5,5\%) Sulfadiazida.

Não foi registrada elevação na média de creatinina entre a primeira e a última dosagem realizadas, entretanto $79(39,5 \%)$ pacientes houve aumento do nível de creatinina enquanto que em 84 (45\%) houve diminuição. Tanto o Clearance de creatinina $(\mathrm{ml} / \mathrm{min})$, como a TFG $(\mathrm{ml} / \mathrm{min} /$ $1,73 \mathrm{~m}^{2}$ ) tiveram uma leve diminuição entre a primeira e a última dosagem, mas ao aplicar o teste não foi encontrada significância estatística, sendo $\mathrm{p}>0,85$ para creatinina sérica, $\mathrm{p}>0,23$ para clearance de creatinina e $\mathrm{p}>0,10$ para TFG.

\section{CONCLUSÃO}

O grupo analisado não mostrou alteração média significativa em relação à creatinina sérica. Porém, foram encontradas alterações metabólicas relacionadas aos lípides, que podem ser possíveis precursoras de doenças cardiovasculares, que por sua vez podem levar à IRC. 
Estudos prospectivos são necessários para melhor controle e acompanhamento do estado clínico, do

\section{REFERÊNCIAS}

1. Brasil. Ministério da Saúde. Boletim Epidemiológico AIDS - Ano IV no 1 - jul a dez de 2006; jan a jun de 2007 [citado 2008 Jun 4]. Disponível em: http://www.AIDS.gov.br/ data/

2. Guimarães MDM. Estudo temporal das doenças associadas à AIDS no Brasil, 1980-1999. Cad Saúde Pública = Rep Public Health. 2000;16(Supl 1):21-36.

3. Sousa AS, Kantorski LP, Bielemann VLM. A AIDS no interior da família: percepcão, silêncio e segredo na convivência social. Acta Sci, Health Sci. 2004;26(1):1-9.

4. Brasil. SBN - Sociedade Brasileira de Nefrologia [Internet]. [citado 2008 Nov 2]. Disponível em: http:// www.sbn.org.br/.

5. National Kidney Foundation K/DOQI clinical practice guidelines for chronic kidney disease: evaluation, classification, and stratification. Am J Kidney Dis. 2002;39(2 Suppl 1):S1-266. tratamento medicamentoso e evolução da função renal destes pacientes.

6. Fine DM. Renal disease and toxicities: issues for HIV care providers. Top HIV Med. 2006;14(5); 164-9. Corpyright 2007, Internatinoal AIDS Society-USA.

7. D'Agati V, Appel GB. HIV infection and the kidney. J Am Soc Nephrol. 1997;8(1):138-52. Review.

8. Ahuja TS, Borucki M, Funtanilla M, Shahinian V, Hollander M, Rajaraman S. Is the prevalence of HIV-associated nephropathy decreasing? Am J Nephrol. 1999;19(6):655-9.

9. Menon V, Gul A, Sarnaki MJ. Cardiovascular risk factors in chronic kidney disease. Kidney Int. 2005;68(4):1413-8. Review.

10. Zoccali C, Mallamaci F, Tripepi G. Traditional and emerging cardiovascular risk factors in end-stage renal disease. Kidney Int Suppl. 2003; 11. Valente AMM, Reis AF, Machado DM, Succi RCM, Chacra AR. Alterações metabólicas da síndrome lipodistrófica do HIV. Arq Bras Endocrinol Metab. 2005;49(6)871-81. 